\title{
Development of An Epi-thermal Neutron Field for Fundamental Researches for BNCT with A DT Neutron Source
}

\author{
Yuta Osawa ${ }^{1,{ }^{\star}, \text { Shoichi Imoto }}{ }^{2}$, Sachie Kusaka ${ }^{1}$, Fuminobu Sato $^{1}$, Masahiro Tanoshita $^{3}$, Isao Murata ${ }^{1}$ \\ ${ }^{1}$ Graduate School of Engineering Osaka University, Yamadaoka 2-1, Suita, Osaka, Japan \\ ${ }^{2}$ City government of Kobe, Kanou 6-5-1, Tyuoku, Kobe, Hyogo, Japan \\ ${ }^{3}$ Department of engineering Fukui University of Technology, Gakuen 3-6-1, Fukui, Fukui, Japan
}

\begin{abstract}
Boron Neutron Capture Therapy (BNCT) is known to be a new promising cancer therapy suppressing influence against normal cells. In Japan, Accelerator Based Neutron Sources (ABNS) are being developed for BNCT. For the spread of ABNS based BNCT, we should characterize the neutron field beforehand. For this purpose, we have been developing a low-energy neutron spectrometer based on ${ }^{3} \mathrm{He}$ position sensitive proportional counter. In this study, a new intense epi-thermal neutron field was developed with a DT neutron source for verification of validity of the spectrometer. After the development, the neutron field characteristics were experimentally evaluated by using activation foils. As a result, we confirmed that an epi-thermal neutron field was successfully developed suppressing fast neutrons substantially. Thereafter, the neutron spectrometer was verified experimentally. In the verification, although a measured detection depth distribution agreed well with the calculated distribution by MCNP, the unfolded spectrum was significantly different from the calculated neutron spectrum due to contribution of the side neutron incidence. Therefore, we designed a new neutron collimator consisting of a polyethylene pre-collimator and boron carbide neutron absorber and confirmed numerically that it could suppress the side incident neutrons and shape the neutron flux to be like a pencil beam.
\end{abstract}

\section{Introduction}

Recently, Boron Neutron Capture Therapy (BNCT) is known to be a new promising cancer therapy. In BNCT, after ${ }^{10} \mathrm{~B}$ is accumulated in cancer cells thermal or epithermal neutrons are irradiated from outside of a human body, and then $\alpha$ and ${ }^{7} \mathrm{Li}$ are produced. These particles have high LET and the ranges are as long as the size of one cell. As a result, tumor cells can selectively be destroyed, simultaneously suppressing influence against normal cells. Figure 1 shows the principle of BNCT.

Up to now, only nuclear reactor has been used as a neutron source for BNCT because BNCT requires a strong low-energy neutron source. In Japan, Accelerator Based Neutron Sources (ABNS) which can be constructed in medical facilities such as hospitals are being developed instead of nuclear reactor. However, characteristics of neutron field such as neutron spectrum and neutron intensity depend on kinds of accelerators or their parameters. Therefore, evaluation of neutron fields obtained in accelerators is important to know and control the effect of therapy, treatment time, and dose of patients. In BNCT, thermal or epi-thermal neutrons are used for the treatment. In particular, estimation of epi-thermal neutron spectrum is known to be difficult because the shape of thermal neutron spectrum is fixed for the atmospheric temperature and fast neutrons can be identified by nuclear reactions such as charged particle emission reactions, e.g., elastic scattering, and activation reactions having threshold energies. For these reasons, a neutron spectrometer is required which can measure thermal and epi-thermal neutron energy and intensity.

In the author's group, a low-energy neutron spectrometer based on a ${ }^{3} \mathrm{He}$ position sensitive proportional counter has been developed [1].

Figure 2 shows the detection principle of the new spectrometer. We measure the detection depth distribution $\mathrm{Y}$ of a nuclear reaction by an incident neutron entering the spectrometer parallel to axis of the neutron detector. We can estimate the neutron energy spectrum X by using an unfolding process with the detector response function $\mathrm{R}$ that was estimated as the reaction depth probability density function for neutron energy.

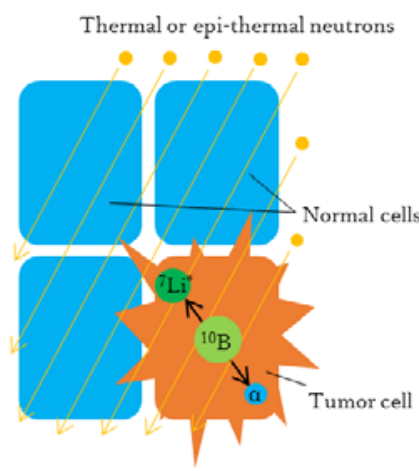

Figure 1 Principle of BNCT.

\footnotetext{
* Corresponding author: y-osawa@qr.see.eng.osaka-u.ac.jp
} 


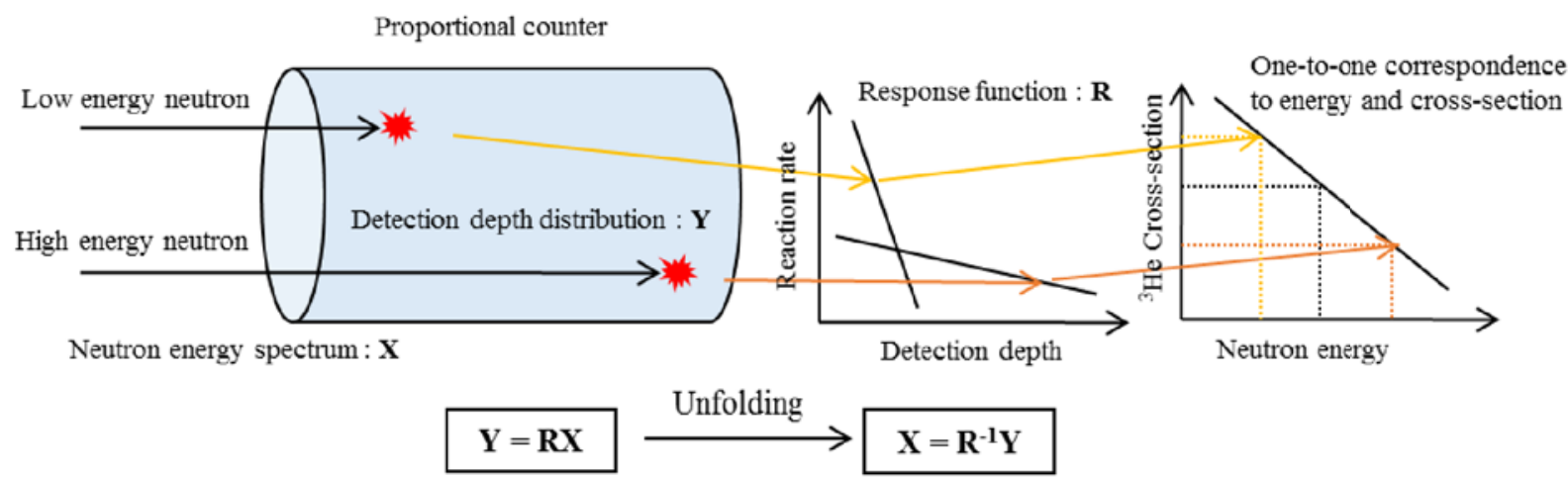

Figure 2 Principle of the neutron spectrometer.

In the previous study, verification of the spectrometer validity has been carried out by using an epi-thermal neutron field made with an AmBe neutron source [2]. However, there was a serious problem we faced, that is, weakness of the utilized AmBe neutron source intensity, leading that the statistical accuracy was seriously deteriorated. As a result, it took a very long time to complete one measurement. Therefore, we developed a new intense epi-thermal neutron field by using the Intense $14 \mathrm{MeV}$ Neutron Source Facility (OKTAVIAN) of Osaka University specifically for fundamental researches of BNCT [3]. After examining the characteristics of the produced neutron field, we have designed and constructed a beam shaping assembly to realize a beam epi-thermal neutron so that we can verify the developed spectrometer experimentally.

\section{Epi-thermal neutron column}

As mentioned in Chapter 1, an intense beam epi-thermal neutron field is required for fundamental researches of BNCT, especially validity verification of the low-energy neutron spectrometer. In this study, we designed and constructed an intense epi-thermal neutron field having a uniform neutron field with as wide range as possible with a DT neutron source instead of an AmBe neutron source. Two design targets of the present epi-thermal neutron field were given as epi-thermal neutron flux $\Phi_{\text {epi-thermal }}$ and ratio of the epi-thermal neutron flux to the fast neutron flux $\eta$. In case of the AmBe neutron source, $\Phi_{\text {epi-thermal }}=$ several tens $\mathrm{n} / \mathrm{sec} / \mathrm{cm}^{2}$ had been achieved by the author's group in the previous study. In this study, hundred times larger epi-thermal neutron flux was aimed, i.e., $\Phi_{\text {epi-thermal }}$ $>1000 \mathrm{n} / \mathrm{sec} / \mathrm{cm}^{2}$, considering the DT neutron source intensity which is several ten-times stronger than the AmBe neutron source. In addition, we defined $\eta$ as $\Phi_{\text {epi- }}$ thermal $/ \Phi_{\text {fast, }}$, which is an index to indicate the mixing degree of fast neutrons in the epi-thermal neutron field. In the present study, the target value of $\eta$ is more than 10 .

\subsection{Design of epi-thermal neutron column}

Design of the epi-thermal neutron field was carried out using Monte Carlo N-Particle Transport Code (MCNP-5) and evaluated nuclear data library of JENDL-4.0 [4]. Differently from the previous design using an $\mathrm{AmBe}$ neutron source $\left(4.0 \times 10^{6} \mathrm{n} / \mathrm{sec}\right)$, the Intense $14 \mathrm{MeV}$ Neutron Source Facility OKTAVIAN of Osaka University was used as a strong neutron source. In the OKTAVIAN Facility, deuteron is accelerated to about $350 \mathrm{keV}$ with a Cockcroft-Walton type electrostatic accelerator and then $14 \mathrm{MeV}$ neutrons are generated by deuteron-triton (DT) reaction. The designed maximum neutron source intensity is around $1.0 \times 10^{9} \mathrm{n} / \mathrm{sec}$ in a pulse mode. In this study, we designed the epi-thermal neutron field as in the following steps under the assumption that the DT neutron intensity was $4.0 \times 10^{8} \mathrm{n} / \mathrm{sec}$ at $5 \mu \mathrm{A}$, which was a common value at present in the facility. Figure 3 shows neutron spectra obtained after each design step.

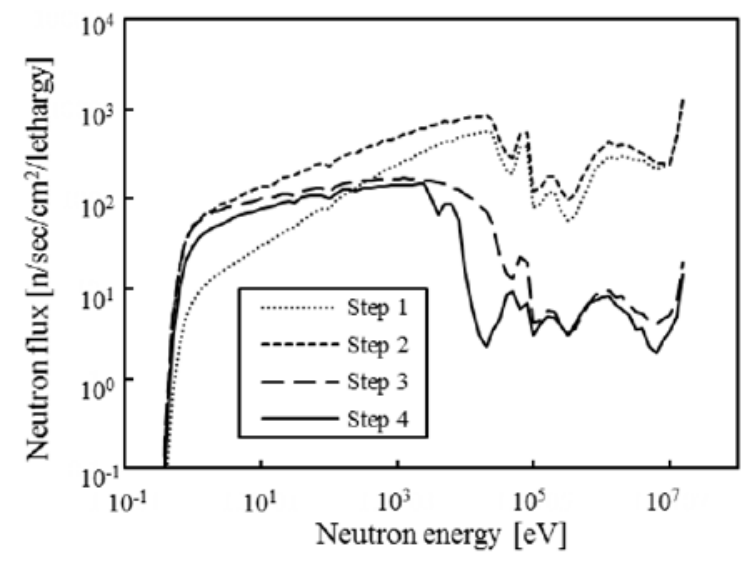

Figure 3 Neutron spectrum for each step.

Table 1 Simuration result of Step 1.

\begin{tabular}{ccc}
\hline Material & $\Phi_{\text {epi-thermal }}\left[\mathrm{n} / \mathrm{sec} / \mathrm{cm}^{2}\right]$ & $\eta$ \\
\hline $\mathrm{Al}$ & 2.1 & 0.00 \\
$\mathrm{AlF}_{3}$ & 5714.7 & 0.58 \\
$\mathrm{C}$ & 3025.3 & 0.20 \\
$\mathrm{CF}_{2}$ & 5487.5 & 0.44 \\
$\mathrm{D}_{2} \mathrm{O}$ & 5515 & 0.55 \\
$\mathrm{Fe}$ & 220.3 & 0.01 \\
$\mathrm{~Pb}$ & 22.9 & 0.00 \\
$\mathrm{PE}$ & 345.7 & 0.07 \\
\hline
\end{tabular}




\subsection{1 $1^{\text {st }}$ moderator of $14 \mathrm{MeV}$ neutrons}

First of all, we selected the first moderator material and determined the moderator thickness for moderating 14 $\mathrm{MeV}$ neutrons effectively (Step 1). The moderator area was $50 \mathrm{~cm} \times 50 \mathrm{~cm}$ seeing from the source and several thick moderators were investigated. As a result, we decided the optimum thickness of $40 \mathrm{~cm}$ which was able to moderate high energy neutrons keeping the epi-thermal neutron flux intensity sufficiently high. In this column, thermal neutrons are not removed by neutron absorbers for other applications. However, thermal neutrons below $0.5 \mathrm{eV}$ could be cut by using a cadmium sheet. Table 1 shows $\Phi_{\text {epi-thermal }}$ of each material we investigated under the same condition. From the result of Table 1, aluminum fluoride shows the best performance as the first moderator. However, because production of an aluminum fluoride block of $50 \mathrm{~cm} \times 50 \mathrm{~cm} \times 40 \mathrm{~cm}$ was technically difficulty, we substituted an aluminum fluoride cylinder of $42 \mathrm{~cm}$ length and $20 \mathrm{~cm}$ in diameter which was successfully produced so far, and the rest space surrounding the cylinder was filled with Teflon pellets to supplement the function of the aluminum fluoride in an actual column.

\subsubsection{Reflector to increase neutron flux intensity}

Next, we determined the thicknesses of side reflectors to increase the neutron flux intensity (Step 2). Lead and graphite, which are known as a good neutron reflector, were selected as candidates. Because normally lead is known to be better than graphite, we examined the performance with lead reflector. As shown in Figure 4, the thicker the lead reflector is, the larger $\Phi_{\text {epi-thermal }}$ becomes. However, $\eta$ saturates for the thickness more than $15 \mathrm{~cm}$. Therefore, we decided the lead thickness was $15 \mathrm{~cm}$. Since lead is much heavier than graphite, we decided to partly combine graphite with lead under the condition that the total thickness of the reflector was $15 \mathrm{~cm}$. From the results of additional analysis shown in Figure 5, combinations of $5 \mathrm{~cm}$ lead and $10 \mathrm{~cm}$ graphite $(\mathrm{Pb}+\mathrm{C}(\mathrm{B}))$ or $10 \mathrm{~cm}$ lead and $5 \mathrm{~cm}$ graphite $(\mathrm{Pb}+\mathrm{C}(\mathrm{C}))$ have similarly

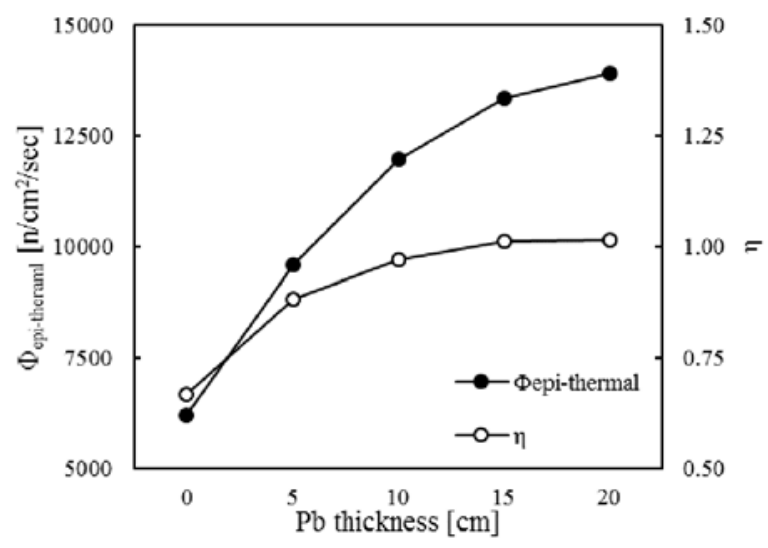

Figure 4 Simulation result of Step 2.

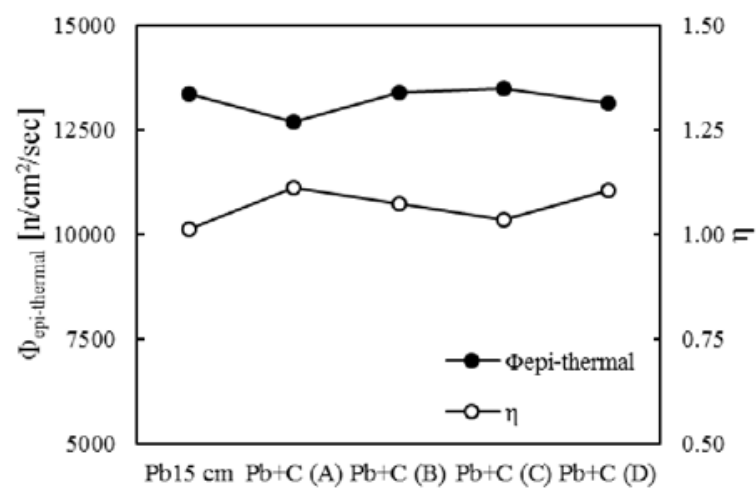

Figure 5 Simulation result of Step 2.

$(\mathrm{Pb}+\mathrm{C}(\mathrm{A})$ : Inside $\mathrm{C} 10 \mathrm{~cm}+$ Outside $\mathrm{Pb} 5 \mathrm{~cm}, \mathrm{~Pb}+\mathrm{C}(\mathrm{B})$ : Inside $\mathrm{Pb} 5 \mathrm{~cm}+$ Outside $\mathrm{C} 10 \mathrm{~cm}, \mathrm{~Pb}+\mathrm{C}(\mathrm{C})$ : Inside $\mathrm{Pb} 10 \mathrm{~cm}+$ Outside C $5 \mathrm{~cm}, \mathrm{~Pb}+\mathrm{C}$ (D): Inside C $5 \mathrm{~cm}+$ Outside $\mathrm{Pb} 10 \mathrm{~cm}$ )

high performance to the $15 \mathrm{~cm}$ lead reflector case. Finally, we decided that the combination of $\mathrm{Pb}+\mathrm{C}$ (B) was employed as the present reflector configuration, because $\mathrm{Pb}+\mathrm{C}(\mathrm{B})$ combination has a larger $\eta$ value and the weight was lighter as compared to $\mathrm{Pb}+\mathrm{C}(\mathrm{C})$ or $\mathrm{Pb} 15 \mathrm{~cm}$ reflector.

\subsubsection{Reduction of fast neutron contribution}

Up to Step 2, as shown in Figure 3, direct contribution of high energy neutrons around $14 \mathrm{MeV}$ neutrons clearly remains. We thus arranged an iron shield as an attenuator in front of the moderator and between the moderator and the neutron source to suppress high energy neutron contribution as small as possible compared to the previous steps (Step 3). Table 2 shows the $\Phi_{\text {epi-thermal }}, \Phi_{14 \mathrm{MeV}}$ and $\eta$ values as a function of the iron shield thickness. The thicker the iron shield becomes, the smaller $\Phi_{14 \mathrm{MeV}}$ is and the larger $\eta$ is, while the $\Phi_{\text {epi-thermal }}$ decreases. In this study, we assumed that both of $\Phi_{\text {epi-thermal }}$ and $\eta$ have equal importance. And then, we made a product of $\Phi_{\text {epi-thermal }}$ and $\eta$ to examine the optimum thickness of the iron shield. The result is shown in Figure 6. From the Figure 6, we determined the thickness of the iron shield attenuator to be $25 \mathrm{~cm}$ because the product showed the highest value.

Table 2 Simulation result of Step 3.

\begin{tabular}{cccc}
\hline $\begin{array}{c}\text { Thickness of iron } \\
{[\mathrm{cm}]}\end{array}$ & $\begin{array}{c}\Phi_{\text {epi-thermal }} \\
{\left[\mathrm{n} / \mathrm{sec}^{2} \mathrm{~cm}^{2}\right]}\end{array}$ & $\begin{array}{c}\Phi_{14 \mathrm{MeV}} \\
{\left[\mathrm{n} / \mathrm{sec}_{\mathrm{cm}}{ }^{2}\right]}\end{array}$ & $\eta$ \\
\hline 0 & 12609.4 & 1276.8 & 0.95 \\
5 & 11844.8 & 534.7 & 1.83 \\
10 & 9976.8 & 238.7 & 2.95 \\
15 & 8060.3 & 106.6 & 4.28 \\
20 & 6363.1 & 42.9 & 5.82 \\
25 & 5036.3 & 19.4 & 7.53 \\
30 & 3984 & 9 & 9.16 \\
35 & 3152.1 & 4.3 & 10.87 \\
40 & 2485.5 & 1.9 & 12.22 \\
45 & 1965.1 & 0.5 & 13.64 \\
50 & 1564.3 & 0.3 & 14.91 \\
\hline
\end{tabular}




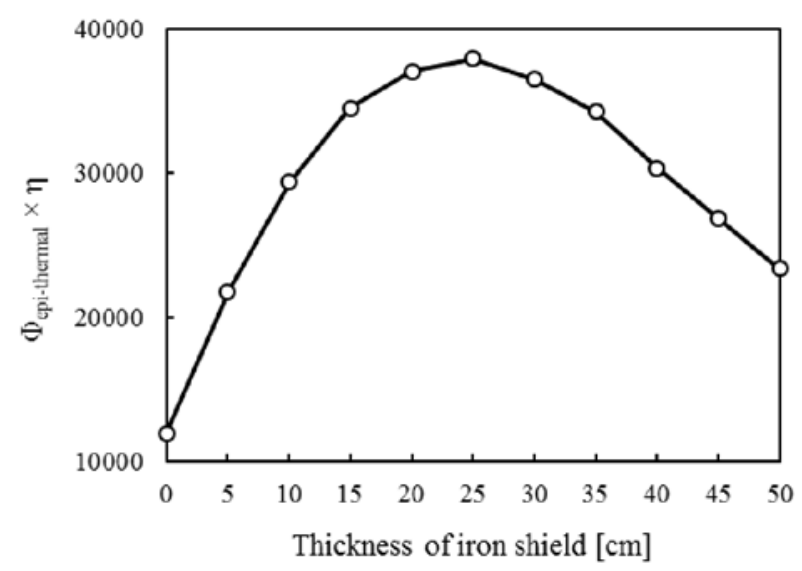

Figure 6 Product of epi-thermal neutro flux and $\eta$.

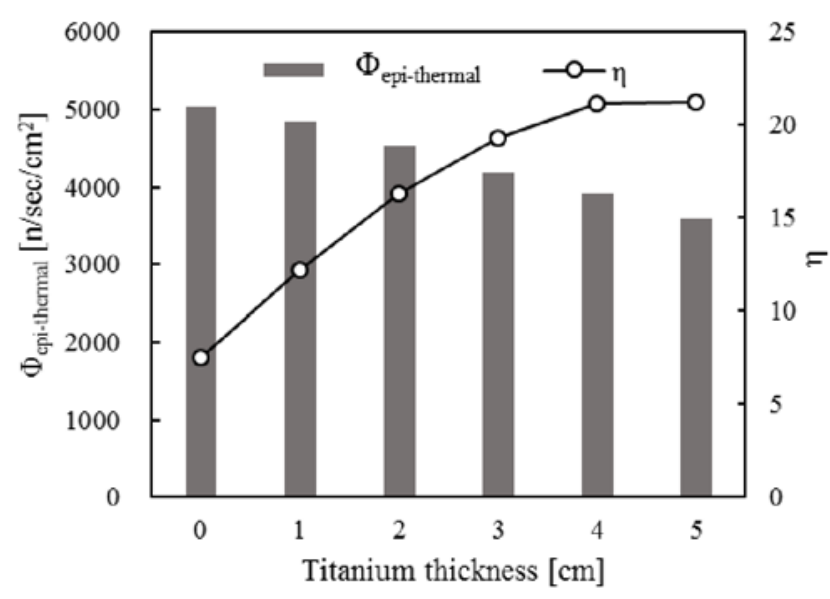

Figure 7 Simulation result of Step 4.

\subsection{4 $2^{\text {nd }}$ moderator to cut higher energy neutrons over $10 \mathrm{keV}$}

Finally, we utilized titanium, which has large resonance above $10 \mathrm{keV}$ region, as the second moderator to cut higher energy neutrons over $10 \mathrm{keV}$ and increase $\eta$ value (Step 4). The size of a titanium plate was $50 \mathrm{~cm} \times 50 \mathrm{~cm}$ and set up in the rear side of the first moderator. We then changed the titanium thickness to decide the optimum thickness. The $\eta$ values saturated over $4 \mathrm{~cm}$ thickness as shown in Figure 7, we thus determined the titanium thickness of $4 \mathrm{~cm}$. However, in the actual epi-thermal neutron column, we used the size of $30 \mathrm{~cm} \times 30 \mathrm{~cm} \times 4 \mathrm{~cm}$ titanium due to its cost.

Figure 8 shows the finally designed neutron column after the 4 steps. From the spectrum after Step 4 in Figure 3 , the designed neutron spectrum has an excellent spectrum shape having a clear peak in the epi-thermal energy region and at the same time supresses fast neutrons very low. Moreover, the designed epi-themal neutron field could accomplish the design target, i.e., $\Phi_{\text {epi-thermal }}=$ $3915 \mathrm{n} / \mathrm{sec} / \mathrm{cm}^{2}(>1000)$ and $\eta=21.2(>10)$. We set this column in a steel container, which is the size of $80 \mathrm{~cm}$ $\times 80 \mathrm{~cm} \times 100 \mathrm{~cm}$ and $700 \mathrm{~kg}$ in weight. Total weight of the column and the container was about $3000 \mathrm{~kg}$.

\subsection{Construction of the epi-thermal neutron column}

The epi-thermal neutron column was constructed by filling designed materials listed in Table 3 in the container of $80 \mathrm{~cm} \times 80 \mathrm{~cm} \times 100 \mathrm{~cm}$. Figure 9 is an inside photo of the column in the middle step of construction. A cylinder of aluminum fluoride moderator was set in the center of an aluminum box. Then Teflon pellets were poured in this box to fill up around the cylinder. Five Iron shield plates and titanium plates were set in the front and rear sides of the box, respectively. Reflector blocks of graphite and lead were arranged so as to cover the moderators and shields entirely.

Table 3 Materials of the epi-thermal neutron column.

\begin{tabular}{ccc}
\hline Material & Size $[\mathrm{cm}]$ & Weight $[\mathrm{kg}]$ \\
\hline $\mathrm{AlF}_{3}$ cylinder & $\varphi 20 \times 42$ & 40.5 \\
$\mathrm{CF}_{2}$ pallets & $50 \times 50 \times 43$ & 115.6 \\
$\mathrm{C}$ blocks & thickness 10 & 470.4 \\
$\mathrm{~Pb}$ blocks & thickness 5 & 900.6 \\
Fe layer & $50 \times 50 \times 25$ & 492.0 \\
Ti plate & $30 \times 30 \times 4$ & 16.2
\end{tabular}

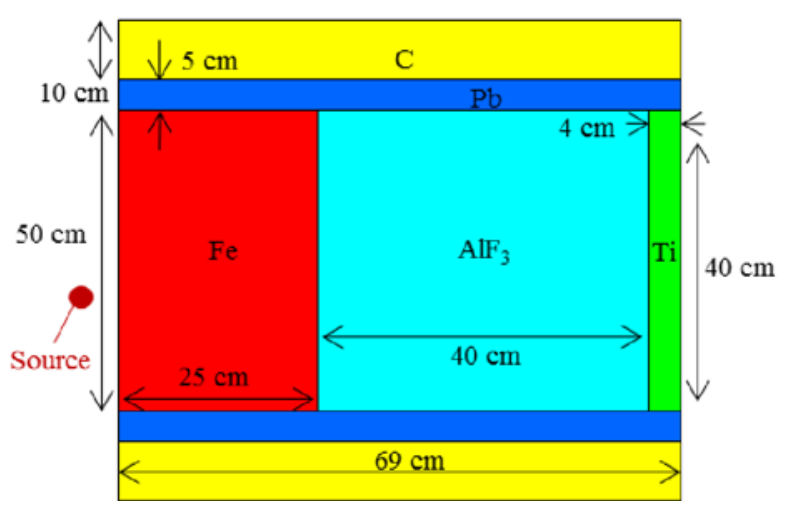

Figure 8 Designed epi-thermal neutron column.

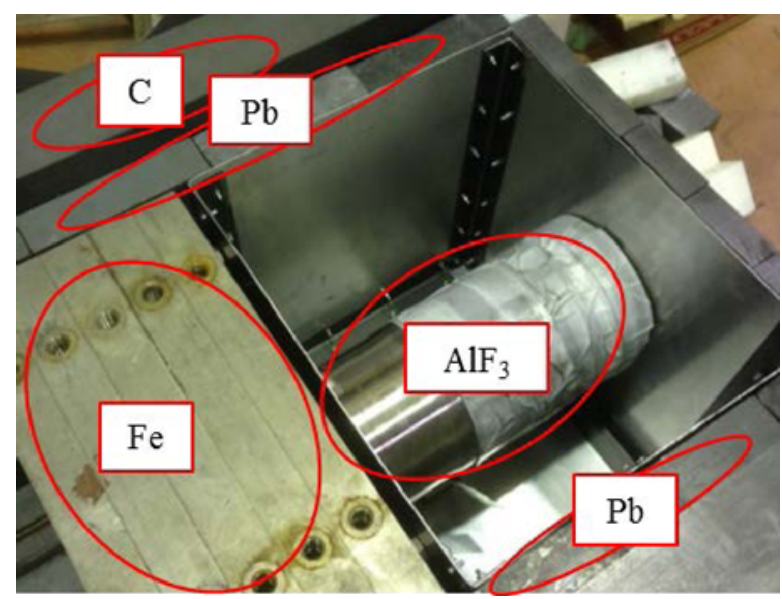

Figure 9 Inside of the epi-thermal neutron column in the middle stage. 


\section{Evaluation of the epi-thermal neutron column}

\subsection{Experiment with activation foils}

For characterization of the constructed epi-thermal neutron column, we have carried out measurements of neutron flux intensity and spectral index with activation foils as shown in Table $4 .{ }^{197} \mathrm{Au}(\mathrm{n}, \gamma){ }^{198} \mathrm{Au}$ reaction is sensitive especially to low energy neutrons. In this experiment, we used gold foils with and without a cadmium absorber and measured the reaction rates to confirm whether the epi-thermal neutron field was formed properly. Indium and aluminum foils were used for an evaluation of the amount of fast neutrons. ${ }^{115} \operatorname{In}\left(\mathrm{n}, \mathrm{n}^{\prime}\right){ }^{115 \mathrm{~m}} \mathrm{In}$ reaction and ${ }^{27} \mathrm{Al}(\mathrm{n}, \alpha)^{24} \mathrm{Na}$ reaction are high threshold energy reactions for $1.2 \mathrm{MeV}$ and $7.2 \mathrm{MeV}$ neutron, respectively. Thus we can examine existence of fast neutrons. Indium foil was covered with a cadmium absorber because ${ }^{115} \mathrm{In}(\mathrm{n}, \gamma){ }^{116 \mathrm{~m}} \mathrm{In}$ reaction has a large cross section with a resonance in the thermal energy region. Figure 10 shows positions of activation foils in this experiment. We set the foils at the same height as the DT neutron source that is $5 \mathrm{~cm}$ lower than the center of the column. In this experiment, the irradiation time was 5 hours and the absolute neutron source intensity was determined using a niobium foil set in front of the neutron source.

\subsection{Experimental result}

The absolute DT neutron intensity was determined to be $1.08 \times 10^{8} \mathrm{n} / \mathrm{sec}$ from a niobium foil in this experiment. After the irradiation, we measured decay gamma-rays of activation foils with two HpGe detectors. Reaction rates of the foils are shown in Table 5 compared with the calculations. Gold foils with and without cadmium were activated sufficiency, and we confirmed that a good agreement was obtained between the calculation and experimental values as shown in the $\mathrm{C} / \mathrm{E}$ values. Next, as for the evaluation of fast neutrons, in the measurement we could not detect gamma-rays from the indium and aluminum foils. It means that higher energy neutron contribution could substantially be suppressed. From these results, we confirmed that an excellent epi-thermal neutron field was constructed having a sufficiently intense epi-thermal neutron flux simultaneously removing fast neutrons.

Table 4 Activation foils used.

\begin{tabular}{cccc}
\hline & Nuclear reaction & $\begin{array}{c}\gamma \text {-ray energy } \\
{[\mathrm{keV}]}\end{array}$ & $\begin{array}{c}\text { Half } \\
\text { life }\end{array}$ \\
\hline${ }^{197} \mathrm{Au}$ & ${ }^{197} \mathrm{Au}(\mathrm{n}, \gamma)^{198} \mathrm{Au}$ & $411.8(95.7 \%)$ & $2.69 \mathrm{~d}$ \\
${ }^{115} \mathrm{In}$ & ${ }^{115} \mathrm{In}\left(\mathrm{n}, \mathrm{n}^{\prime}\right){ }^{115 \mathrm{~m}} \mathrm{In}$ & $336.3(45.8 \%)$ & $4.49 \mathrm{~h}$ \\
${ }^{27} \mathrm{Al}$ & ${ }^{27} \mathrm{Al}(\mathrm{n}, \alpha)^{24} \mathrm{Na}$ & $1369(100 \%)$ & $14.7 \mathrm{~h}$ \\
\hline
\end{tabular}

Table 5 Comparison of experiment and calculation.

\begin{tabular}{cccc}
\hline $\begin{array}{c}\text { Activation } \\
\text { foil }\end{array}$ & $\begin{array}{c}\text { Reaction rate } \\
\text { (calculation) } \\
{[/ \text { sec/source }]}\end{array}$ & $\begin{array}{c}\text { Reaction rate } \\
\text { (experiment) } \\
{[/ \text { sec/source }]}\end{array}$ & $\mathrm{C} / \mathrm{E}$ \\
\hline \multirow{2}{*}{$\mathrm{Au}$} & $2.67 \times 10^{-6}$ & $2.48 \times 10^{-6}$ & 1.08 \\
& $( \pm 3.94 \%)$ & $( \pm 2.14 \%)$ & \\
$\mathrm{Au}+\mathrm{Cd}$ & $2.28 \times 10^{-6}$ & $2.17 \times 10^{-6}$ & 1.05 \\
& $( \pm 4.08 \%)$ & $( \pm 3.59 \%)$ & \\
$\mathrm{In}$ & $2.97 \times 10^{-9}$ & Not detected & \\
& $( \pm 13.2 \%)$ & & \\
$\mathrm{Al}$ & $5.50 \times 10^{-10}$ & Not detected & \\
& $( \pm 23.4 \%)$ & &
\end{tabular}

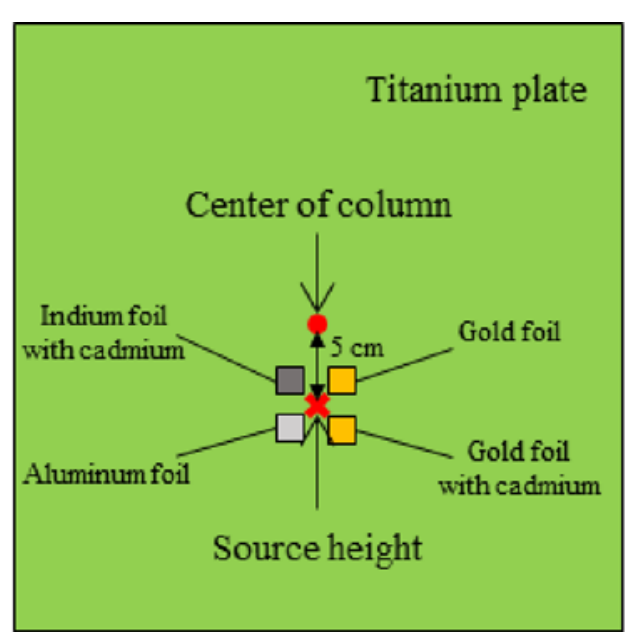

Figure 10 Setting position pf activation foils.

\section{Experimental verification of the low energy neutron spectrometer}

\subsection{Principle of detection}

There is no direct method to measure the low energy neutron spectrum. In the author's group, a new lowenergy neuron spectrometer was developed using a position sensitive ${ }^{3} \mathrm{He}$ proportional counter [2]. The principle is as follows: ${ }^{3} \mathrm{He}$ has a large reaction-cross section in lower energy regions, in addition, the crosssection monotonically increases as the velocity of neutron deceases. Thus it shows a clear one-to-one correspondence between neutron energy and ${ }^{3} \mathrm{He}$ crosssection value. Therefore, when neutrons are incident to the spectrometer in parallel to the axis of the neutron detector, we will measure many nuclear reactions near the entrance of the detector because low-energy neutrons have a large reaction cross-section. By contrast, higherenergy neutrons will be measured at deeper positions. Namely, if we measure a neutron detection depth distribution in the detector, the distribution is expected to change depending on the neutron energy. The reaction depth probability density function for neutron energy can thus be used as the detector response function to estimate the neutron energy. The response matrix R(E,r)dr can ea- 


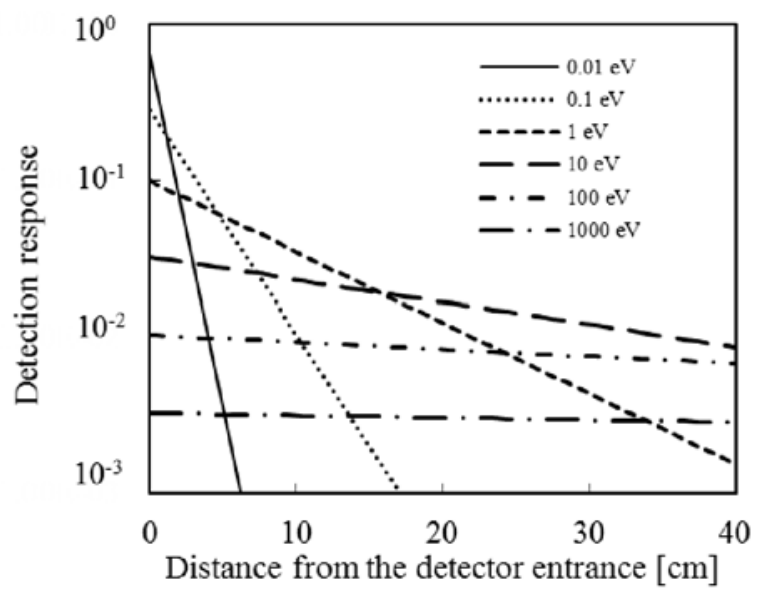

Figure 11 Response function of detector: R(E,r). Each line corresponds to incident neutron energy from $0.01 \mathrm{eV}$ to $1000 \mathrm{eV}$.

sily be deduced as $\sum(E) \exp \left(-\sum(E) r\right) \cdot d r$ if neglecting the effect of a casing, where $\sum(\mathrm{E})$ is macroscopic cross section of ${ }^{3} \mathrm{He}(n, p)$ reaction, $E$ is neutron energy and $r$ is depth. Figure 11 illustrates some examples of the detector response function for the presently used ${ }^{3} \mathrm{He}$ detector. If measured detection depth distribution is $\mathrm{Y}(\mathrm{r})$ and neutron energy spectrum is $\mathrm{X}(\mathrm{E})$, they are related by $\mathrm{Y}(\mathrm{r})=$ $\int R(E, r) X(E) d E$. The neutron energy spectrum is estimated by using unfolding process from the measured reaction position distribution, $\mathrm{Y}(\mathrm{r})$. For the unfolding process, we adopted the Bayes theorem [5].

\subsection{Detector}

Figure 12 shows the ${ }^{3} \mathrm{He}$ position sensitive proportional counter we used in this study. The length is $40 \mathrm{~cm}$, the diameter of the detector is $2.5 \mathrm{~cm}$ and the ${ }^{3} \mathrm{He}$ gas pressure is $0.5 \mathrm{MPa}$. The core wire resistance we used as anode is $4.6 \mathrm{k} \Omega$. The position sensitive proportional counter has two output BNC connectors at both ends to identify where the incident neutron reacts with inner gaseous material. When the emitted charged particles induce secondary electrons in the counter and they reach the anode, the induced charge is separated and conveyed to two directions and different voltage pulses are generated according to the reaction position because of a high resistance level of the core wire. Two output signals are amplified adequately by two pairs of pre-amplifiers and main-amplifiers, and then fed to a multi-parameter system (MPS-1600). Figure 13 shows the block diagram of the measurement circuit. The measurable energy range of this proportional counter was estimated to be from $0.01 \mathrm{eV}$ to $1 \mathrm{keV}$ in the previous study [6].

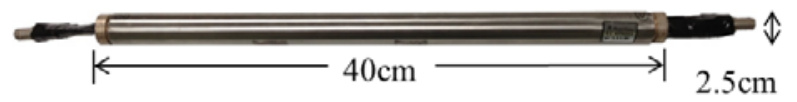

Figure $12{ }^{3} \mathrm{He}$ position sensitive proportional counter.

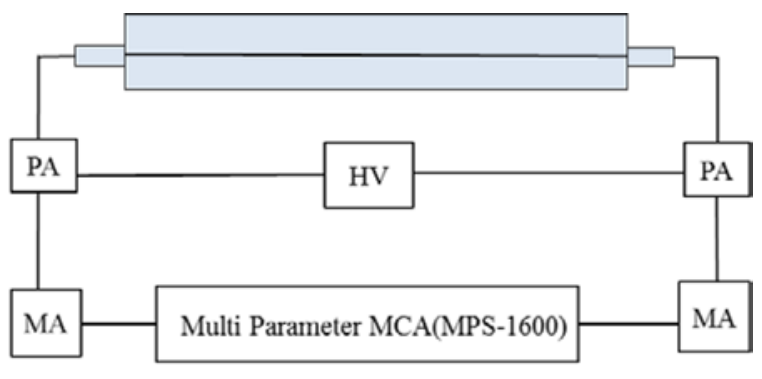

PA: Preamplifier (703-1C, OKEN)

HV: High voltage power supply (714-1E, OKEN)

MA: Amplifier (590A, ORTEC)

Figure 13 Schematic block diagram of measuring system.

\subsection{Verification}

\subsubsection{Experiment}

We carried out experimental verification of the neutron spectrometer with the epi-thermal neutron column designed and constructed in Chapter 2. Figure 14 shows the experimental setup. The DT neutron source was positioned at $8.5 \mathrm{~cm}$ distant from the column and shifted $5 \mathrm{~cm}$ vertically and $2.3 \mathrm{~cm}$ horizontally from the center of the column. The detector was set on the deuteron beam axis and at $20 \mathrm{~cm}$ away from the column. From the standpoint of the detection principle of the present neutron spectrometer, incident neutrons should be incident in parallel to the axis of the detector, that is, the neutron beam shaping is required. We covered the detector using cadmium sheet as a neutron absorber and polyethylene as a neutron moderator to collimate neutrons and to suppress influence of neutrons entering from the side of the detector. Practically, the neutrons entering from the side was moderated to thermal neutrons by polyethylene and cadmium can cut thermal neutrons. Accordingly, we shaped the neutron flux of the epi-thermal neutron column to be a neutron beam. The irradiation time was 5 hours and we used a niobium foil to determine the absolute neutron source intensity by setting it in front of the neutron source.

\subsubsection{Result}

In the experiment, the absolute DT neutron source intensity was determined to be $8.9 \times 10^{8} \mathrm{n} / \mathrm{sec}$ from a niobium foil. Figure 15 shows the measured detection depth distribution. In the distribution, there was no count at both ends of the detector, because proportional counter has dead regions at the both ends. From the result of the detection depth distribution, the count number was large near the entrance of the detector and was smaller at deeper positions. It means that low energy neutrons entered the detector from one side of it and then much more neutrons reacted with internal ${ }^{3} \mathrm{He}$ gas near the detector entrance. In Figure 15, the calculated detection position distribution by MCNP-5 was shown. From the figure, it was found that the calculation result agreed with the measurement result very well. However, the unfolded neutron spectrum was significantly different from the calculated spectrum by 


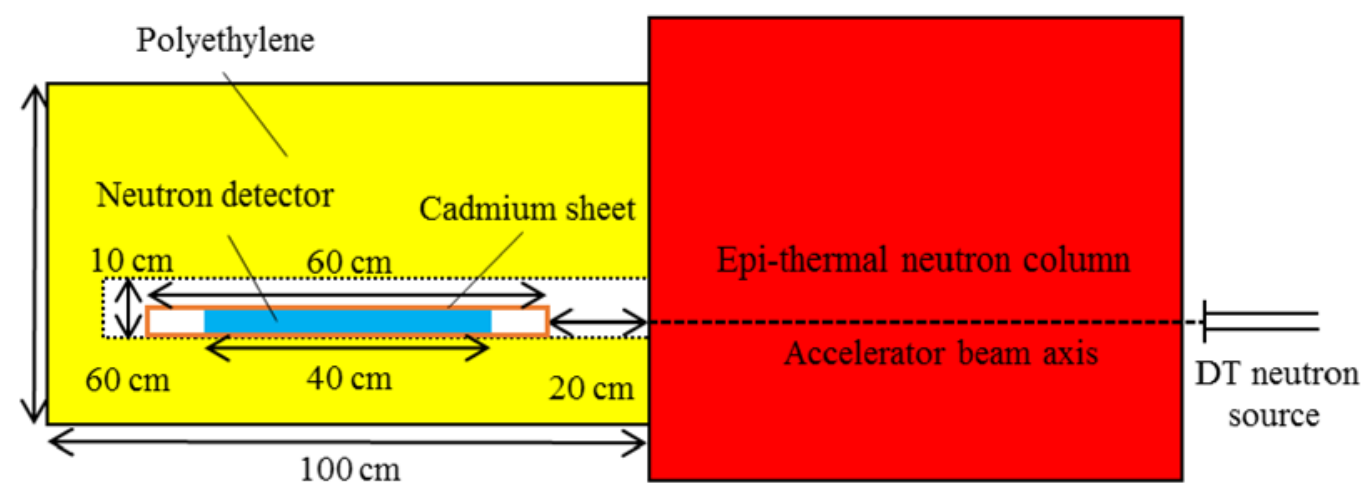

Figure 14 Experimental setup.

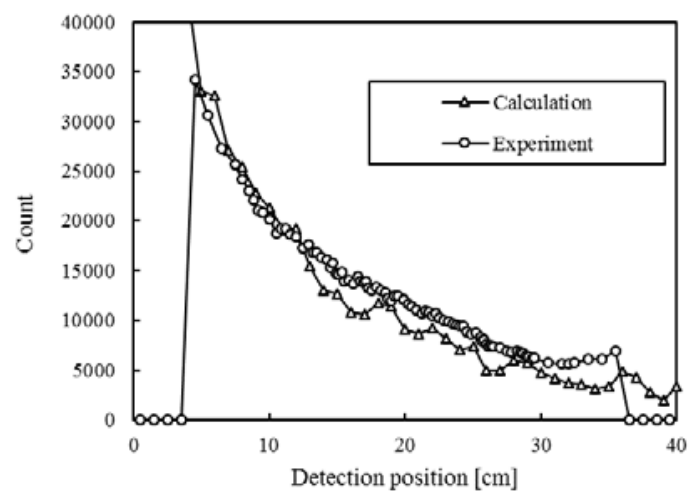

Figure 15 Detction depth distribution.

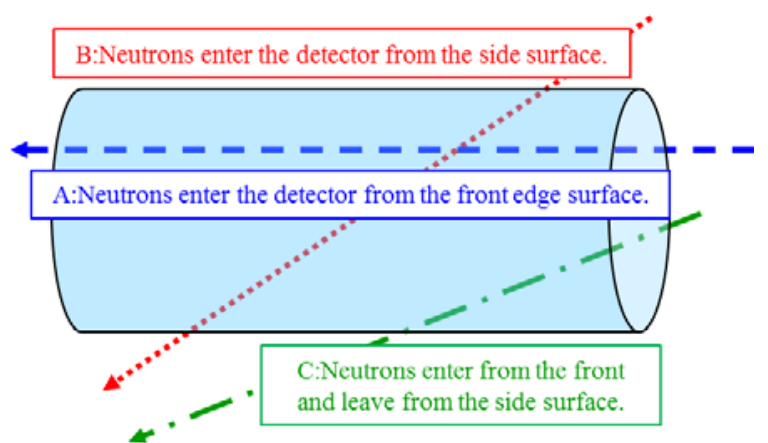

Figure 16 Incident neutrons.

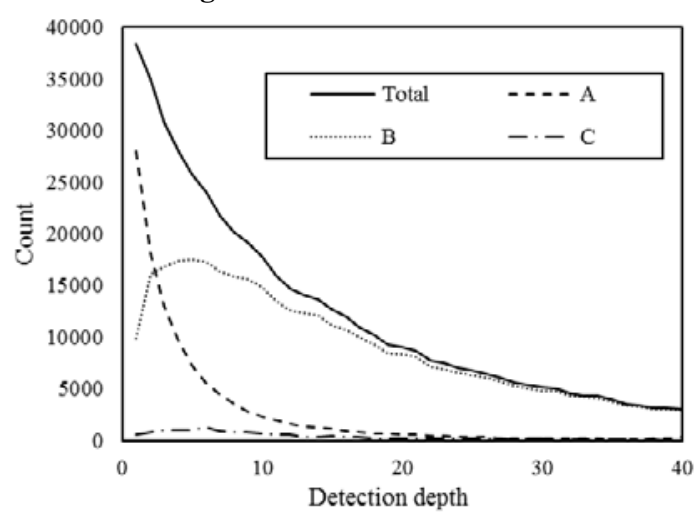

Figure 17 Detection depth distribution.

Total: $\mathrm{A}+\mathrm{B}+\mathrm{C}$, calculation result in Figure 15, A: Neutrons enter the detector from the front edge surface, B: Neutrons enter from the side surface, and C: Neutrons enter from the front and leave from the side surface.
MCNP. To examine the discrepancy, we analyzed the contribution of incident neutrons to the detector separately, resolving the incident neutrons into three components; A, B and $\mathrm{C}$ as shown in Figure 16. As a result of Figure 17, it is made clear that not incident neutrons from the front edge (A) but those from the side surface (B) dominantly cause the detection depth distribution of. Namely, the cadmium sheet and polyethylene moderator could not suppress neutron's entering from the side of the detector sufficiently.

\section{Design of a new neutron collimator}

The experimental result of Chapter 4 suggested that a spectrometer shield having a collimator role made of cadmium and polyethylene should be improved. It is expected that it can shape the neutron flux to be a clear angular flux and at the same time to shield neutron incidence from the side of the detector, in order to carry out the experimental verification of the present neutron spectrometer. In this chapter, we design a new neutron collimator consisting of a pre-collimator and neutron shields.

\subsection{Design of pre-collimator}

To collimate the neutron flow, we set a pre-collimator additionally in front of the detector as show in Figure 18. The performance of pre-collimator saturated over $20 \mathrm{~cm}$. Therefore, the length of the pre-collimator was determined with $20 \mathrm{~cm}$ because the intensity was the highest in the thickness more than $20 \mathrm{~cm}$ and the diameter was $4 \mathrm{~cm}$. We investigated suitable materials by examining angular distributions of neutron incident angles to the detector entrance surface as shown in Figure 19. Borated polyethylene or polyethylene pre-collimator could shape the neutron flux to be like a pencil beam. Although both of them have almost the same performance, we decided to use polyethylene in consideration of costs. 


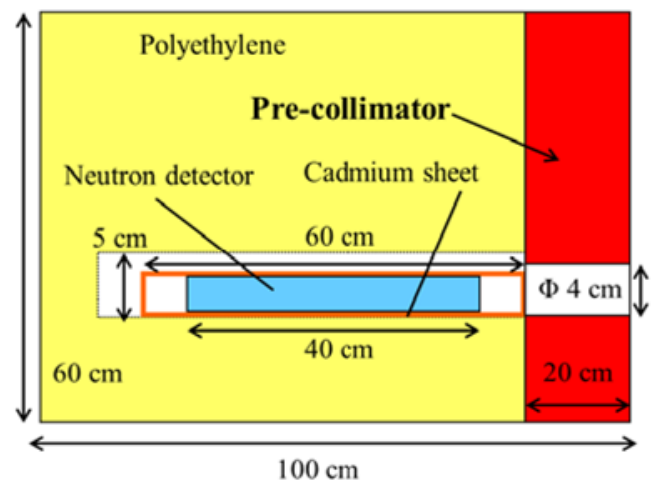

Figure 18 Pre-collimator design.

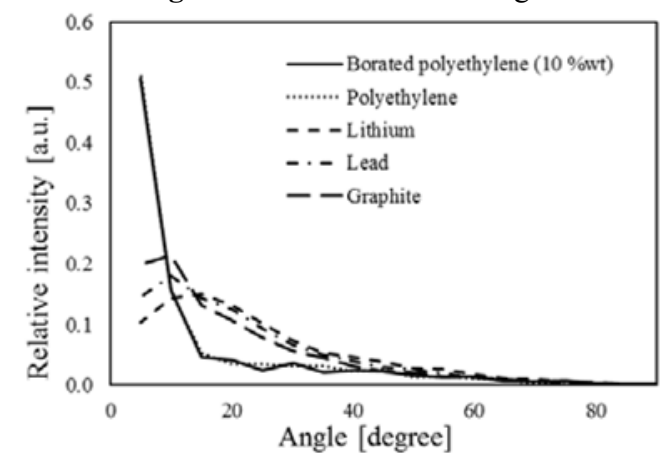

Figure 19 Angular distribution of neutrons incident to the detector entrance surface for several materials.

\subsection{Design of neutron shield}

For suppressing the side incident neutrons, we installed a neutron absorber surrounding the neutron detector in addition to a polyethylene layer covering the neutron shield as a neutron moderator as shown in Figure 20. We selected lithium and solid boron carbide as the neutron absorber material because these materials are known as a good neutron absorber, so that we could cut neutrons for energies smaller than the cadmium cutoff energy of $0.5 \mathrm{eV}$. In order to determine the material and thickness, we changed their thickness and analyzed the contribution of incident neutrons; A, B and C in Figure 16. Figure 21 shows each contribution at $20 \mathrm{~cm}$ depth from the detector entrance. As shown in Figure 21, the performance of lithium becomes greatest at $20 \mathrm{~cm}$ thick, while the performance of solid boron carbide saturates over $5 \mathrm{~mm}$. Comparing $20 \mathrm{~cm}$ lithium and $5 \mathrm{~mm}$ boron carbide, boron carbide has better performance. Therefore, we decided to use boron carbide of $5 \mathrm{~mm}$ thick for the neutron shield.

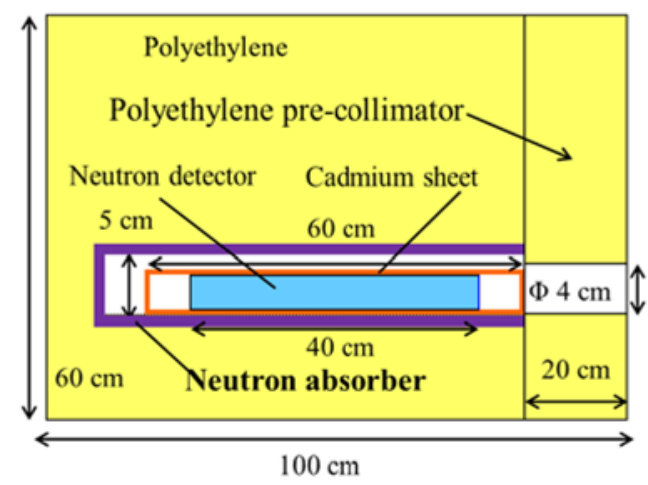

Figure 20 Neutron shield design around the detector.

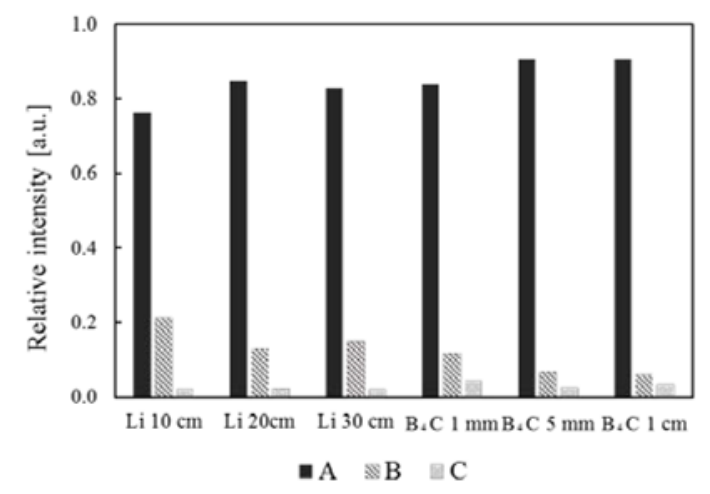

Figure 21 Performance of neutron absorbers of $\mathrm{Li}$ or $\mathrm{B}_{4} \mathrm{C}$.

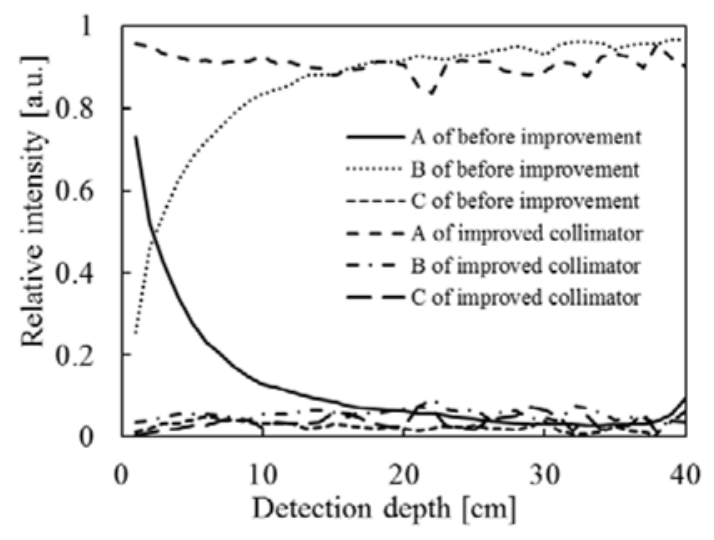

Figure 22 Presently obtained collimator performance.

Finally, the performance of the designed neutron collimator is shown in Figure 22. The designed neutron collimator could suppress influence of the side incident neuron to be sufficiency low and shape the neutron flux to be an excellent pencil beam.

\section{Conclusion}

We designed and constructed an epi-thermal neutron field with a DT neutron source and then evaluated the characteristics of the produced neutron field by using activation foils. Additionally, the experimental verification of the developed neutron spectrometer was carried out. As a result of the evaluation of the neutron field, we confirmed the epi-thermal neutron field was successfully formed so as to supply a high epi-thermal neutron flux field simultaneously suppressing fast neutrons substantially. The present neutron field is expected to give an epi-thermal flux intensity of over $1 \times 10^{4} \mathrm{n} / \mathrm{sec} / \mathrm{cm}^{2}$ and would be available for fundamental researches of BNCT, if the OKTAVIAN facility provides the spec value of $1 \times 10^{9} \mathrm{n} / \mathrm{sec}$. In the experimental verification of the neutron spectrometer, the measured detection depth distribution agreed well with the calculation. However, the unfolded neutron spectrum was different from the neutron spectrum predicted by MCNP due to contribution of neutron incidence from the side surface of the detector. It means that the present beam shaping assembly with cadmium and polyethylene should be improved in order appropriately to carry out the 
experimental verification of the neutron spectrometer. Therefore, we designed a new neutron collimator consisting of polyethylene pre-collimator and boron carbide neutron absorber with a polyethylene shield, so that it can shape the neutron flux to be an excellent pencil beam suppressing neutron incidence from the side surface of the detector substantially.

\section{References}

[1] I. Murata, H.Miyamaru, Low-energy neutron spectrometer using position sensitive proportional counter-Feasibility study based on numerical analysis, Nuclear Instruments and methods in Physics Research A 589,445-454(2008).

[2] K. Tsubouchi, Design calculation of epi-thermal neutron field with DT neutron source for low energy neutron spectrometer developed for BNCT, Master's thesis, Osaka University (2014) [in Japanese].

[3] K. Sumita, A.Takahashi, T. Iida, J. Yamamto, S. Imoto, K. Matuda, Osaka University 14Mev Intense Neutron Source and Its Utilizations for Fusion Studies (OKTAVIAN Program), Fusion Technology 1, 675-680 (1982)

[4] X-5 Monte Carlo Team, MCNP-A General Monte Carlo N-Particle Transport Code Vesion 5, (2003).

[5] S. Iwasaki, A new approach for unfolding PHA problems based only the Bayes' Theorem, Proc. 9 th International Symposium on Rector Dosimetry, Prague, Chezk Republic, 245-252 (1996).

[6] T. Obata, Development of keV region neutron spectrometer using 3 He proportional counter, Master's thesis, Osaka University (2013) [in Japanese] 